\title{
Oscillation Sensitivity with Upward-going Muons in ICAL at India-based Neutrino Observatory (INO)
}

\author{
R. Kanishka ${ }^{* a}$, Vipin Bhatnagar ${ }^{a}$ and D. Indumathi ${ }^{b}$ \\ ${ }^{a}$ Panjab University, Chandigarh 160014, India \\ ${ }^{b}$ The Institute of Mathematical Sciences, Chennai 600 113, India \\ E-mail: kanishka.rawat.phy@gmail.com, vipin@pu.ac.in, \\ indu@imsc.res.in
}

\begin{abstract}
The proposed magnetised Iron Calorimeter (ICAL) detector at the India-based Neutrino Observatory (INO) lab is mostly sensitive to the atmospheric muon neutrinos. These are detected through the detection of charged muons, arising from the charged-current (CC) interactions of muonneutrinos with the material of the detector, and are the primary signal in the study of atmospheric neutrinos. Upward-going muons also known as rock muons, arise from the interactions of atmospheric neutrinos with the rock material surrounding the detector in the earth's crust. We present the oscillation sensitivity results with upward-going muons and discuss their significance in INO.
\end{abstract}

16th International Workshop on Neutrino Factories and Future Neutrino Beam Facilities - NUFACT2014, 25 -30 August, 2014

University of Glasgow, United Kingdom

*Speaker. 


\section{Introduction}

Upward-going or rock muons [1] [2] provide an independent measurement of the oscillation parameters. An independent measurement will further provide a consistency check with the contained vertex analysis and would result in slight improvement of the overall measurement. Though the sensitivity of upward-going muons to the oscillation parameters is lower than contained vertex events, even then the analysis is helpful in any neutrino experiment. Here we show some preliminary results on upward-going muons. A comparison with Super-K results is also done for the consistency. In this paper, we present the oscillation sensitivity with upward-going muons in ICAL [3] using the resolutions and efficiencies of the peripheral and side region (mainly bottom part) [4] of the ICAL detector.

Iron CALorimeter (ICAL) consists of three identical modules of dimension $16 \mathrm{~m} \times 16 \mathrm{~m} \times 14.45$ $\mathrm{m}$, with 151 layers of $5.6 \mathrm{~cm}$ thick magnetized iron plates interleaved by Resistive Plate Chambers (RPCs) [5]. The RPCs are active detector elements and have good position and time resolution. The magnetic field which is generated by passing current through copper coils, which pass through coil slots in the plates as shown in Fig. 1, is distributed non-uniformly in such a way that it divides the whole ICAL (each module) in three main regions. The main region is "central region" [7]; within which the coils slots has the highest, and uniform $\left(B_{y}\right)$ magnetic field. The region in which the field is about $15 \%$ smaller and in the opposite direction to the central region, is labelled as the "side region" (outside the coil slots in the $x$ direction). The region labelled as "peripheral region" [4] (outside the central region in the $y$ direction) has the changing magnetic field in both direction and magnitude. Hence, both the side and peripheral regions will be affected by edge effects i.e., both of them have partially contained events. So, the resolutions and efficiencies in peripheral and side region are worse than the central region. Alongwith them, there is one right and left side of the detector, both of them contains resolution of the side region. Three front and back of the detector contains resolution of the peripheral region whereas the three bottom part of the ICAL has the resolution which contains average of the resolutions of all the regions. Our study includes resolutions and efficiencies generated at the three bottom part of the detector.

The paper is organised as follows: we will discuss the production of upward-going muons in ICAL detector in next subsection. We discuss the methodology in section 2 and oscillation results in section 3 . We conclude with discussions in the section 4.

\subsection{Upward-Going Muons at ICAL Detector}

Upward-going muons basically arise from the interactions of atmospheric neutrinos with the rock material surrounding the detector, and it carries the signature of oscillation. But as the muon energy increases, its survival probability $\left(P_{\mu \mu}\right)$ (given by eq. (1.1) ) goes to one.

$$
P_{\mu \mu}=1-\sin ^{2} 2 \theta_{23} \sin ^{2}\left(1.27 \times \Delta m_{32}^{2} \times \frac{L}{E}\right)
$$

where, $\theta_{23}=45^{\circ}, \Delta m_{32}^{2}=2.4 \times 10^{-3} \mathrm{eV}^{2}$

Fig. 2 shows the production of upward-going muons at ICAL. Neutrinos after interacting with rock produce hadrons [8] which gets absorbed in the rock, and upward-going muons, which travels 


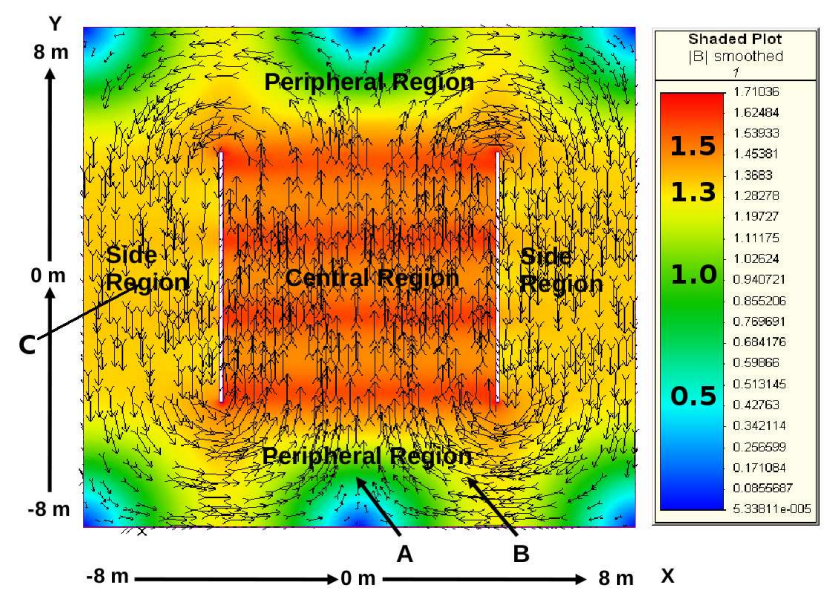

Fig. 1: Magnetic field map as generated by the MAGNET6 [6] software. Here, A: $(x=0, y=-650, z=0) \mathrm{cm}$, B: $(x=300, y=-650, z=0) \mathrm{cm}, C:(x=-2270, y=0, z=0) \mathrm{cm}$; actually located in the left-most module.

the distance $\mathrm{L}$ and makes angle $\theta$ with the $\mathrm{z}$ - axis w.r.t ICAL, then finally reaches the ICAL detector. Due to its production mechanism, upward-going muons are to be discriminated from two kind of events: the neutrino events producing muons through the interactions inside ICAL detector, which comes under the main studies of ICAL. This can be eliminated by taking muon track which comes from outside the ICAL detector. Secondly, cosmic ray muon events produced in the earth's atmosphere directly interacting with ICAL; these are the main background of the ICAL detector. This can be eliminated by putting an angle cut (discussed in the next section), which only allows upward-going muons for the analysis.

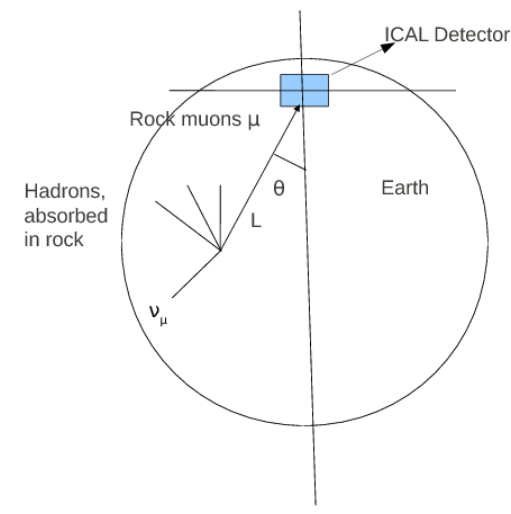

Fig. 2: Production of upward-going muons at ICAL.

Muon loses most of the energy in the rock before it reaches detector so the oscillation signature becomes more complicated. Though their number is reduced due to neutrinos undergoing oscilla- 
tions and also due to energy loss mechanisms, even then they are significant for ICAL detector.

Fig. 3 shows the linear relationship in the energy of muons $E_{\mu}$, calculated from formula (eq. (1.3)) vs. $E_{\mu}$ from Nuance [9] (neutrino generator) data at the detector. The eq. (1.2) shows the average muon energy loss formula for muons of energy $E_{\mu}$ in the rock.

$$
\frac{d E_{\mu}}{d x}=-a-b E_{\mu}
$$

The eq. (1.3) shows energy loss of the muons after the propagation of $X \mathrm{~g} / \mathrm{sq} . \mathrm{cm}$ depth.

$$
E_{\mu}=\left(E_{\mu}^{0}+e\right) \exp (-b X)-e
$$

where, $E^{0}$ is the initial muon energy, $a$ accounts for ionization losses and $b$ accounts for the three radiation processes: bremsstrahlung, production of electron-positron pairs and photoproduction. We have, $e=a / b=500 \mathrm{GeV}$.

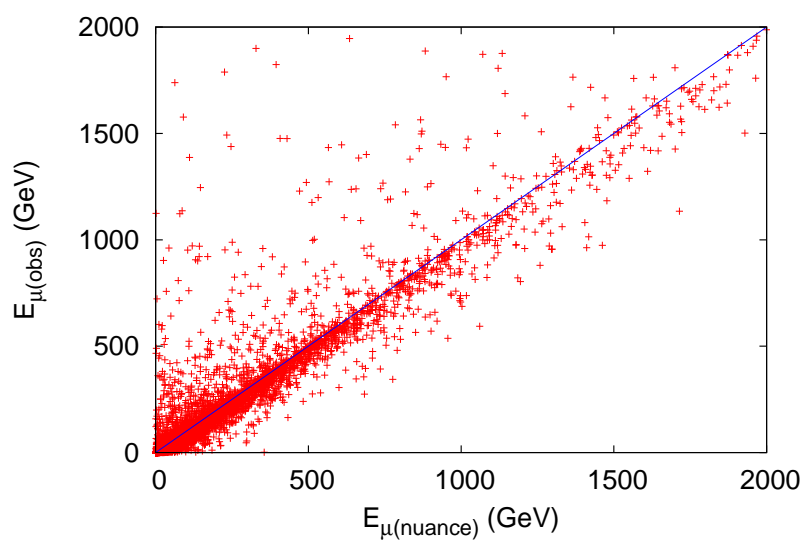

Fig. 3: $E_{\mu}$ calculated from formula vs. $E_{\mu}$ from Nuance data at the detector.

In the next section, we discuss the methodology to study upward-going muons.

\section{Methodology}

Here we discuss the methodology adopted to generate the data and the contour in 2-dimensional parameter space $\left(\sin ^{2} 2 \theta_{23}, \Delta m_{32}^{2}\right)$ for upward-going muons. 200 years data is generated using NuanceV3.504 (neutrino generator). Input parameters taken for data generation are $\theta_{23}=45^{\circ}\left(\sin \theta_{23}\right.$ $=0.707), \Delta m^{2}=2.4 \times 10^{-3} \mathrm{eV}^{2}, \theta_{12}=34^{\circ}, \theta_{13}=0^{\circ}, \delta_{c p}=0$. Dimension of the detector is taken such that no events are generated inside the detector; only its external geometry is required and the actual material in which interactions happen is rock, whose density is taken to be $2.65 \mathrm{gm} / \mathrm{cc}$. To cut off cosmic ray backgrounds, a cut on angle was applied which is $0^{\circ}<\theta<70^{\circ}(\cos \theta>0.342)$. We took only CC $v_{\mu}$ events for the analysis which were not passed through ICAL detector (Geant 4 [10] based INO-ICAL code[7]). Then, muon energy and angle is smeared according to the look-up tables of upward-going muons which includes resolutions and efficiencies of the bottom part of the whole ICAL detector. Data is oscillated using 2 - flavor formula (eq. (1.1)) and binned into energy 
and $\cos \theta$ bins. The generated data sample has proportionately larger component of higher energy events which are not sensitive to the oscillations, so we have taken finer bins at lower energy. Energy range is taken from 1 to $256 \mathrm{GeV} / \mathrm{c}$. The $\cos \theta$ bins are taken as: $0.2-0.4,0.4-0.6,0.6-0.8$, 0.8-1. Fig. 4 shows the survival probability $P_{\mu \mu}$ for $\theta_{13}=0^{\circ}$. Here, figure shows that the probability goes to unity at higher energy, hence its not sensitive and therefore, we took fine bins at low energy.

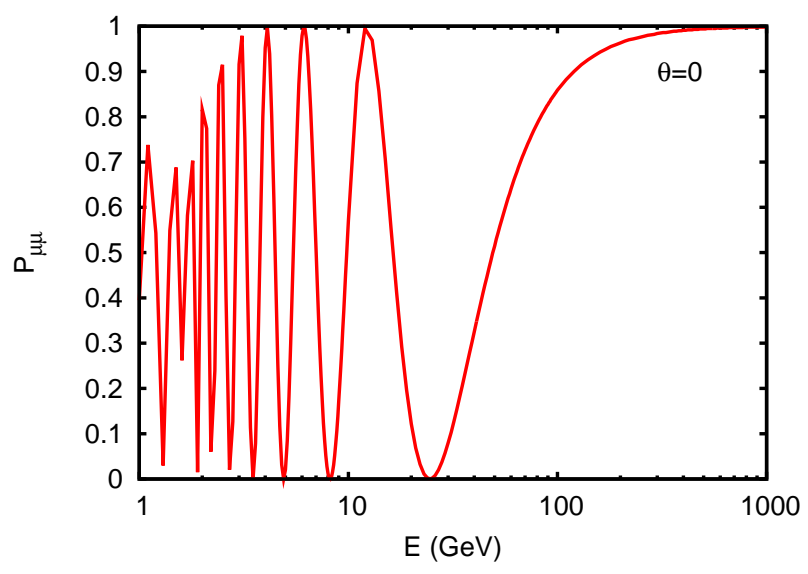

Fig. 4: Survival probability $P_{\mu \mu}$ for $\theta_{13}=0^{\circ}$ (generated from Nuance data).

Then, upward-going muon data is scaled down to 4.5 years to have a comparison with Super-K results.

The $\chi^{2}$ definition with pull method; that takes into account systematic errors, is used in this analysis. Only one pull is used for our analysis, and $\chi^{2}$ definition is given by the eq. (2.1) :

$$
\chi^{2}=\sum_{i}\left(\frac{N_{i}^{t h}(1+\pi \zeta)-N_{i}^{e x}}{\sigma_{i}^{s t a t}}\right)^{2}+\zeta^{2}
$$

Where, the various parameters are:

- $N_{i}^{t h}, N_{i}^{e x}=$ theoretically predicted data and observed data.

- $\pi=$ systematic errors taken; normalisation error of $20 \%$, no tilt factor included.

- $\zeta=$ Pull variable with respect to which $\chi^{2}$ is minimized.

- $\sigma_{i}^{\text {stat }}=$ Statistical errors, defined as $\sqrt{ }\left(N_{i}^{e x}\right)$.

We have plotted $90 \%$ CL contours for the two parameters; $\sin ^{2} 2 \theta_{23}, \Delta m_{32}^{2}$ with $\Delta \chi^{2}=4.61$ definition, and its shown in the next section. Our analysis does not include any background events. Charge identification (cid) efficiency is not taken, as we have combined $\mu^{-}$with $\mu^{+}$events. We will show the final results based on all the inputs, resolutions and efficiencies, in the next section. 


\section{Oscillations Results}

The energy distribution of muon $\left(E_{\mu}\right)$ for different $\cos \theta$ bins is shown in Fig. 5. It is noticed that in first $\cos \theta$ bin i.e., $0.2-0.4$ there are just few events, as we have put an angle cut of $\cos \theta>$ 0.342 to exclude cosmic ray background. Since, the events get oscillated at lower energy hence, there is fine binning at lower energy but not at higher energy (reason as explained from Fig. 4).
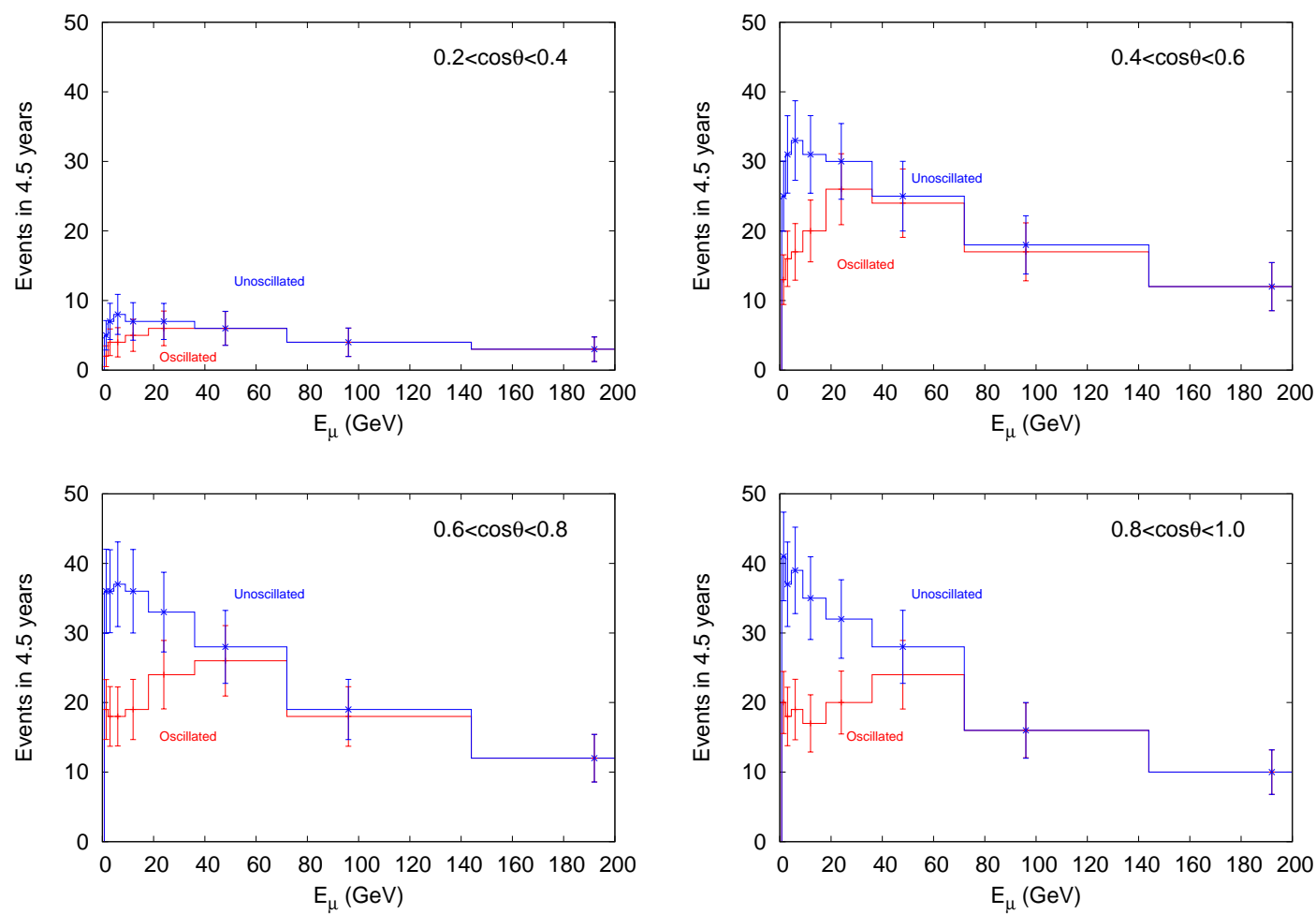

Fig. 5: Energy distribution of muon $\left(E_{\mu}\right)$ for different $\cos \theta$ bins.

Fig. 6 shows $90 \%$ CL contour of ICAL for 4.5 years and is compared with Super-K [11] for consistency. For Super-K: $\sin ^{2} 2 \theta_{23}=0.765, \Delta m_{32}^{2}=(1.2-4.3) \times 10^{-3} \mathrm{eV}^{2}$. For ICAL $: \sin ^{2} 2 \theta_{23}=$ $0.77, \Delta m_{32}^{2}=(1.48-3.7) \times 10^{-3} \mathrm{eV}^{2}$, whereas, from $\mathrm{CC}$ atmospheric muon neutrino events (main ICAL studies with contained vertex events) [12]: $\sin ^{2} 2 \theta_{23}=0.979, \Delta m_{32}^{2}=(2.1-2.9) \times 10^{-3} \mathrm{eV}^{2}$. From both the parameters, the sensitivity is comparable for ICAL and Super-K with upward-going muons.

\section{Discussions and Conclusion}

The ICAL detector at India-based Neutrino Observatory is a proposed underground facility to study the oscillation pattern of atmospheric neutrinos, precise measurement of oscillation parameters, probing neutrino mass hierarchy as well as new physics. An independent measurement of the oscillation parameters is provided by upward-going or rock muons [13] which would improve the overall measurement, though the sensitivity of upward-going muons to the oscillation parameters is lower than the contained vertex events. Upward-going muon comes from the interaction 


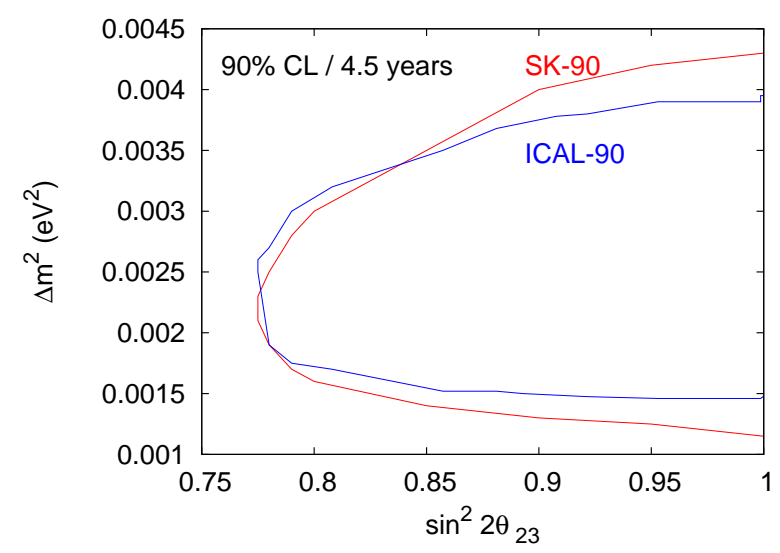

Fig. 6: $90 \%$ CL contour of ICAL and Super-K in 4.5 years of data.

of atmospheric neutrinos with the rock material surrounding the detector, and carries the signature of oscillation. For this analysis, resolutions and efficiencies were taken for the events going from inside the bottom part of the detector. $90 \% \mathrm{CL}$ contour was obtained for ICAL for 4.5 years of data which gives $\sin ^{2} 2 \theta_{23}=0.77, \Delta m_{32}^{2}=(1.48-3.7) \times 10^{-3} \mathrm{eV}^{2}$. These results are compared with Super-K data also, and the sensitivity for upward-going muons is comparable for both ICAL and Super-K detector. The ICAL reach in 10 years will be more informative and will help in the better understanding, and the work related to it is going on.

Acknowledgements : We thank INO collaboration for the help and support. We also thank the INO Physics group coordinators for their comments and suggestions. R. Kanishka acknowledges UGC/DST (Govt. of India) for financial support.

\section{References}

[1] Y. Fukuda et al., Super-Kamiokande Collaboration, Measurement of the flux and zenith-angle distribution of upward through-going muons by Super-Kamiokande, Phys. Rev. Lett. 82 (1999) 2644, [arXiv:9812014v2].

[2] Y. Ashie et al., Super-Kamiokande Collaboration, A Measurement of Atmospheric Neutrino Oscillation Parameters by Super-Kamiokande-I, Phys. Rev. D 71 (2005) 112005, [arXiv:0501064v2].

[3] M. S. Athar et al., 2006 India-based Neutrino Observatory: Project Report Volume I, http://www.ino.tifr.res.in/ino/OpenReports/INOReport.pdf.

[4] R. Kanishka et al., Simulations Study of Muon Response in the Peripheral Regions of the Iron Calorimeter Detector at the India-based Neutrino Observatory, submitted to JINST, 2014.

[5] B. Satyanarayana, Design and Characterisation Studies of Resistive Plate Chambers, PhD thesis, Department of Physics, IIT Bombay, PHY-PHD-10-701, (2009).

[6] Infolytica Corp., Electromagnetic field simulation software, http://www.infolytica.com/en/products/magnet/.

[7] A. Chatterjee et al., A Simulations Study of the Muon Response of the Iron Calorimeter detector at the India-based Neutrino Observatory, 2014 JINST 9 P07001, [arXiv:1405.7243]. 
[8] M. M. Devi et al., Hadron energy response of the Iron Calorimeter detector at the India-based Neutrino Observatory, 2013 JINST 8 P11003 [arXiv:1304.5115].

[9] D. Casper, The Nuance neutrino physics simulation, and the future, Nucl. Phys. Proc. Suppl. 112 (2002) 161-170, [arXiv:0208030].

[10] GEANT4 collaboration, S. Agostinelli et al. Geant4: a simulation toolkit, Nucl. Instrum. Meth. A 506 (2003) 250-303; http://geant4.cern.ch.

[11] Kazunori Nitta, Neutrino Oscillation Analysis of Upward Through-going and Stopping Muons in Super-Kamiokande, PhD thesis, Department of Physics, Osaka University, 2003 http://www-sk.icrr.u-tokyo.ac.jp/sk/pub/nitta.pdf

[12] T. Thakore, A. Ghosh, S. Choubey and A. Dighe, The Reach of INO for Atmospheric Neutrino Oscillation Parameters, JHEP 1305, (2013) 058 [arXiv:1303.2534].

[13] M. Ambrosio et al., The MACRO Collaboration, Measurement of the atmospheric neutrino-induced upgoing muon flux using MACRO, Phy. Lett. B 434, (1998) 451-457, [arXiv:9807005v1] 